\title{
Evolution of Cannibalistic Traits: Scenarios Derived from Adaptive Dynamics
}

Fabio Dercole (dercole@elet.polimi.it)

Sergio Rinaldi (rinaldi@elet.polimi.it)

\section{Approved by}

Ulf Dieckmann (dieckman@iiasa.ac.at)

Project Leader, Adaptive Dynamics Network

July 2002 


\section{IIASA STUDIES IN ADAPTIVE DYNAMICS No. 66}

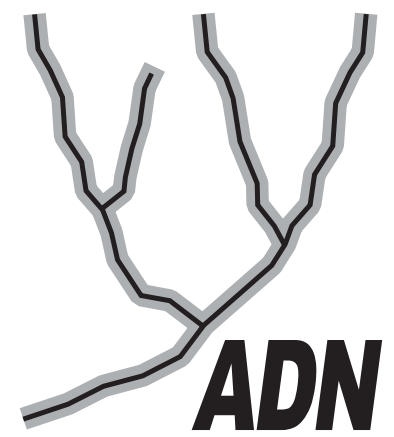

The Adaptive Dynamics Network at IIASA fosters the development of new mathematical and conceptual techniques for understanding the evolution of complex adaptive systems.

Focusing on these long-term implications of adaptive processes in systems of limited growth, the Adaptive Dynamics Network brings together scientists and institutions from around the world with IIASA acting as the central node.

Scientific progress within the network is collected in the IIASA Studies in Adaptive Dynamics series.

No. 1 Metz JAJ, Geritz SAH, Meszéna G, Jacobs FJA, van Heerwaarden JS: Adaptive Dynamics: A Geometrical Study of the Consequences of Nearly Faithful Reproduction. IIASA Working Paper WP-95-099 (1995). van Strien SJ, Verduyn Lunel SM (eds): Stochastic and Spatial Structures of Dynamical Systems, Proceedings of the Royal Dutch Academy of Science (KNAW Verhandelingen), North Holland, Amsterdam, pp. 183-231 (1996).

No. 2 Dieckmann U, Law R: The Dynamical Theory of Coevolution: A Derivation from Stochastic Ecological Processes. IIASA Working Paper WP-96-001 (1996). Journal of Mathematical Biology 34:579-612 (1996).

No. 3 Dieckmann U, Marrow P, Law R: Evolutionary Cycling of Predator-PreyInteractions: Population Dynamics and the Red Queen. IIASA Preprint (1995). Journal of Theoretical Biology 176:91-102 (1995).

No. 4 Marrow P, Dieckmann U, Law R: Evolutionary Dynamics of Predator-Prey Systems: An Ecological Perspective. IIASA Working Paper WP-96-002 (1996). Journal of Mathematical Biology 34:556-578 (1996).

No. 5 Law R, Marrow P, Dieckmann U: On Evolution under Asymmetric Competition. IIASA Working Paper WP-96-003 (1996). Evolutionary Ecology 11:485-501 (1997).

No. 6 Metz JAJ, Mylius SD, Diekmann O: When Does Evolution Optimize? On the Relation Between Types of Density Dependence and Evolutionarily Stable Life History Parameters. IIASA Working Paper WP-96-004 (1996).

No. 7 Ferrière R, Gatto M: Lyapunov Exponents and the Mathematics of Invasion in Oscillatory or Chaotic Populations. Theoretical Population Biology 48:126-171 (1995).

No. 8 Ferrière R, Fox GA: Chaos and Evolution. IIASA Preprint (1996). Trends in Ecology and Evolution 10:480485 (1995)

No. 9 Ferrière R, Michod RE: The Evolution of Cooperation in Spatially Heterogeneous Populations. IIASA Working Paper WP-96-029 (1996). The American Naturalist 147:692717 (1996).

No. 10 van Dooren TJM, Metz JAJ: Delayed Maturation in Temporally Structured Populations with Non-Equilibrium Dynamics. IIASA Working Paper WP-96-070 (1996). Journal of Evolutionary Biology 11:41-62 (1998).
No. 11 Geritz SAH, Metz JAJ, Kisdi É, Meszéna G: The Dynamics of Adaptation and Evolutionary Branching. IIASA Working Paper WP-96-077 (1996). Physical Review Letters 78:2024-2027 (1997).

No. 12 Geritz SAH, Kisdi É, Meszéna G, Metz JAJ: Evolutionary Singular Strategies and the Adaptive Growth and Branching of the Evolutionary Tree. IIASA Working Paper WP-96-114 (1996). Evolutionary Ecology 12:35-57 (1998).

No. 13 Heino M, Metz JAJ, Kaitala V: Evolution of Mixed Maturation Strategies in Semelparous Life-Histories: The Crucial Role of Dimensionality of Feedback Environment. IIASA Working Paper WP-96-126 (1996). Philosophical Transactions of the Royal Society of London Series B 352:1647-1655 (1997).

No. 14 Dieckmann U: Can Adaptive Dynamics Invade? IIASA Working Paper WP-96-152 (1996). Trends in Ecology and Evolution 12:128-131 (1997).

No. 15 Meszéna G, Czibula I, Geritz SAH: Adaptive Dynamics in a 2-Patch Environment: A Simple Model for Allopatric and Parapatric Speciation. IIASA Interim Report IR-97-001 (1997). Journal of Biological Systems 5:265-284 (1997).

No. 16 Heino M, Metz JAJ, Kaitala V: The Enigma of Frequency-Dependent Selection. IIASA Interim Report IR97-061 (1997). Trends in Ecology and Evolution 13:367-370 (1998).

No. 17 Heino M: Management of Evolving Fish Stocks. IIASA Interim Report IR-97-062 (1997). Canadian Journal of Fisheries and Aquatic Sciences 55:1971-1982 (1998).

No. 18 Heino M: Evolution of Mixed Reproductive Strategies in Simple Life-History Models. IIASA Interim Report IR-97063 (1997).

No. 19 Geritz SAH, van der Meijden E, Metz JAJ: Evolutionary Dynamics of Seed Size and Seedling Competitive Ability. IIASA Interim Report IR-97-071 (1997). Theoretical Population Biology 55:324-343 (1999).

No. 20 Galis F, Metz JAJ: Why Are There So Many Cichlid Species? On the Interplay of Speciation and Adaptive Radiation. IIASA Interim Report IR-97-072 (1997). Trends in Ecology and Evolution 13:1-2 (1998). 
No. 21 Boerlijst MC, Nowak MA, Sigmund K: Equal Pay for all Prisoners/ The Logic of Contrition. IIASA Interim Report IR-97-073 (1997). American Mathematical Society Monthly 104:303-307 (1997). Journal of Theoretical Biology 185:281-293 (1997).

No. 22 Law R, Dieckmann U: Symbiosis Without Mutualism and the Merger of Lineages in Evolution. IIASA Interim Report IR-97-074 (1997). Proceedings of the Royal Society of London Series B 265:1245-1253 (1998).

No. 23 Klinkhamer PGL, de Jong TJ, Metz JAJ: Sex and Size in Cosexual Plants. IIASA Interim Report IR-97-078 (1997). Trends in Ecology and Evolution 12:260-265 (1997).

No. 24 Fontana W, Schuster P: Shaping Space: The Possible and the Attainable in RNA Genotype-Phenotype Mapping. IIASA Interim Report IR-98-004 (1998). Journal of Theoretical Biology 194:491-515 (1998).

No. 25 Kisdi É, Geritz SAH: Adaptive Dynamics in Allele Space: Evolution of Genetic Polymorphism by Small Mutations in a Heterogeneous Environment. IIASA Interim Report IR-98-038 (1998). Evolution 53:993-1008 (1999).

No. 26 Fontana W, Schuster P: Continuity in Evolution: On the Nature of Transitions. IIASA Interim Report IR-98-039 (1998). Science 280:1451-1455 (1998).

No. 27 Nowak MA, Sigmund K: Evolution of Indirect Reciprocity by Image Scoring/ The Dynamics of Indirect Reciprocity. IIASA Interim Report IR-98-040 (1998). Nature 393:573-577 (1998). Journal of Theoretical Biology 194:561574 (1998).

No. 28 Kisdi É: Evolutionary Branching Under Asymmetric Competition. IIASA Interim Report IR-98-045 (1998). Journal of Theoretical Biology 197:149-162 (1999).

No. 29 Berger U: Best Response Adaptation for Role Games. IIASA Interim Report IR-98-086 (1998).

No. 30 van Dooren TJM: The Evolutionary Ecology of Dominance-Recessivity. IIASA Interim Report IR-98-096 (1998). Journal of Theoretical Biology 198:519-532 (1999).

No. 31 Dieckmann U, O'Hara B, Weisser W: The Evolutionary Ecology of Dispersal. IIASA Interim Report IR-98-108 (1998). Trends in Ecology and Evolution 14:88-90 (1999).

No. 32 Sigmund K: Complex Adaptive Systems and the Evolution of Reciprocation. IIASA Interim Report IR-98-100 (1998). Ecosystems 1:444-448 (1998).

No. 33 Posch M, Pichler A, Sigmund K: The Efficiency of Adapting Aspiration Levels. IIASA Interim Report IR-98103 (1998). Proceedings of the Royal Society London Series B 266:1427-1435 (1999).

No. 34 Mathias A, Kisdi É: Evolutionary Branching and Coexistence of Germination Strategies. IIASA Interim Report IR-99-014 (1999).

No. 35 Dieckmann U, Doebeli M: On the Origin of Species by Sympatric Speciation. IIASA Interim Report IR-99-013 (1999). Nature 400:354-357 (1999).

No. 36 Metz JAJ, Gyllenberg M: How Should We Define Fitness in Structured Metapopulation Models? Including an Application to the Calculation of Evolutionarily Stable Dispersal Strategies. IIASA Interim Report IR-99-019 (1999). Proceedings of the Royal Society of London Series B 268:499508 (2001)
No. 37 Gyllenberg M, Metz JAJ: On Fitness in Structured Metapopulations. IIASA Interim Report IR-99-037 (1999). Journal of Mathematical Biology 43:545-560 (2001).

No. 38 Meszéna G, Metz JAJ: Species Diversity and Population Regulation: The Importance of Environmental Feedback Dimensionality. IIASA Interim Report IR-99-045 (1999).

No. 39 Kisdi É, Geritz SAH: Evolutionary Branching and Sympatric Speciation in Diploid Populations. IIASA Interim Report IR-99-048 (1999).

No. 40 Ylikarjula J, Heino M, Dieckmann U: Ecology and Adaptation of Stunted Growth in Fish. IIASA Interim Report IR-99-050 (1999). Evolutionary Ecology 13:433-453 (1999).

No. 41 Nowak MA, Sigmund K: Games on Grids. IIASA Interim Report IR-99-038 (1999). Dieckmann U, Law R, Metz JAJ (eds): The Geometry of Ecological Interactions: Simplifying Spatial Complexity, Cambridge University Press, Cambridge, UK, pp. 135-150 (2000).

No. 42 Ferrière R, Michod RE: Wave Patterns in Spatial Games and the Evolution of Cooperation. IIASA Interim Report IR-99-041 (1999). Dieckmann U, Law R, Metz JAJ (eds): The Geometry of Ecological Interactions: Simplifying Spatial Complexity, Cambridge University Press, Cambridge, UK, pp. 318-332 (2000).

No. 43 Kisdi É, Jacobs FJA, Geritz SAH: Red Queen Evolution by Cycles of Evolutionary Branching and Extinction. IIASA Interim Report IR-00-030 (2000).

No. 44 Meszéna G, Kisdi É, Dieckmann U, Geritz SAH, Metz JAJ: Evolutionary Optimisation Models and Matrix Games in the Unified Perspective of Adaptive Dynamics. IIASA Interim Report IR-00-039 (2000).

No. 45 Parvinen K, Dieckmann U, Gyllenberg M, Metz JAJ: Evolution of Dispersal in Metapopulations with Local Density Dependence and Demographic Stochasticity. IIASA Interim Report IR-00-035 (2000).

No. 46 Doebeli M, Dieckmann U: Evolutionary Branching and Sympatric Speciation Caused by Different Types of Ecological Interactions. IIASA Interim Report IR-00-040 (2000). The American Naturalist 156:S77-S101 (2000).

No. 47 Heino M, Hanski I: Evolution of Migration Rate in a Spatially Realistic Metapopulation Model. IIASA Interim Report IR-00-044 (2000). The American Naturalist 157:495511 (2001).

No. 48 Gyllenberg M, Parvinen K, Dieckmann U: Evolutionary Suicide and Evolution of Dispersal in Structured Metapopulations. IIASA Interim Report IR-00-056 (2000). Journal of Mathematical Biology 45:79-105 (2002).

No. 49 van Dooren TJM: The Evolutionary Dynamics of Direct Phenotypic Overdominance: Emergence Possible, Loss Probable. IIASA Interim Report IR-00-048 (2000). Evolution 54: 1899-1914 (2000).

No. 50 Nowak MA, Page KM, Sigmund K: Fairness Versus Reason in the Ultimatum Game. IIASA Interim Report IR00-57 (2000). Science 289:1773-1775 (2000).

No. 51 de Feo O, Ferrière R: Bifurcation Analysis of Population Invasion: On-Off Intermittency and Basin Riddling. IIASA Interim Report IR-00-074 (2000). International Journal of Bifurcation and Chaos 10:443-452 (2000).

No. 52 Heino M, Laaka-Lindberg S: Clonal Dynamics and Evolution of Dormancy in the Leafy Hepatic Lophozia Silvicola. IIASA Interim Report IR-01-018 (2001). Oikos 94:525-532 (2001). 
No. 53 Sigmund K, Hauert C, Nowak MA: Reward and Punishment in Minigames. IIASA Interim Report IR-01-031 (2001). Proceedings of the National Academy of Sciences of the USA 98:10757-10762(2001).

No. 54 Hauert C, De Monte S, Sigmund K, Hofbauer J: Oscillations in Optional Public Good Games. IIASA Interim Report IR-01-036 (2001).

No. 55 Ferrière R, Le Galliard J: Invasion Fitness and Adaptive Dynamics in Spatial Population Models. IIASA Interim Report IR-01-043 (2001). Clobert J, Dhondt A, Danchin E, Nichols J (eds): Dispersal, Oxford University Press, pp. 57-79 (2001).

No. 56 de Mazancourt C, Loreau M, Dieckmann U: Can the Evolution of Plant Defense Lead to Plant-Herbivore Mutualism. IIASA Interim Report IR-01-053 (2001). The American Naturalist 158: 109-123 (2001).

No. 57 Claessen D, Dieckmann U: Ontogenetic Niche Shifts and Evolutionary Branching in Size-Structured Populations. IIASA Interim Report IR-01-056 (2001). Evolutionary Ecology Research 4:189-217 (2002).

No. 58 Brandt H: Correlation Analysis of Fitness Landscapes. IIASA Interim Report IR-01-058 (2001).

No. 59 Dieckmann U: Adaptive Dynamics of Pathogen-Host Interacations. IIASA Interim Report IR-02-007 (2002). Dieckmann U, Metz JAJ, Sabelis MW, Sigmund K (eds): Adaptive Dynamics of Infectious Diseases: In Pursuit of Virulence Management, Cambridge University Press, Cambridge, UK, pp. 39-59 (2002).

No. 60 Nowak MA, Sigmund K: Super- and Coinfection: The Two Extremes. IIASA Interim Report IR-02-008 (2002).
Dieckmann U, Metz JAJ, Sabelis MW, Sigmund K (eds): Adaptive Dynamics of Infectious Diseases: In Pursuit of Virulence Management, Cambridge University Press, Cambridge, UK, pp. 124-137 (2002).

No. 61 Sabelis MW, Metz JAJ: Perspectives for Virulence Management: Relating Theory to Experiment. IIASA Interim Report IR-02-009 (2002). Dieckmann U, Metz JAJ, Sabelis MW, Sigmund K (eds): Adaptive Dynamics of Infectious Diseases: In Pursuit of Virulence Management, Cambridge University Press, Cambridge, UK, pp. 379-398 (2002).

No. 62 Cheptou P, Dieckmann U: The Evolution of SelfFertilization in Density-Regulated Populations . IIASA Interim Report IR-02-024 (2002). Proceedings of the Royal Society of London Series B 269:1177-1186 (2002).

No. 63 Bürger R: Additive Genetic Variation Under Intraspecific Competition and Stabilizing Selection: A Two-Locus Study. IIASA Interim Report IR-02-013 (2002). Journal of Theoretical Population Biology 61:197-213 (2002).

No. 64 Hauert C, De Monte S, Hofbauer J, Sigmund K: Volunteering as Red Queen Mechanism for Co-operation in Public Goods Games. IIASA Interim Report IR-02-041 (2002). Science 296:1129-1132 (2002).

No. 65 Dercole F, Ferrière R, Rinaldi S: Ecological Bistability and Evolutionary Reversals under Asymmetrical Competition. IIASA Interim Report IR-02-053 (2002). Evolution 56:1081-1090 (2002).

No. 66 Dercole F, Rinaldi S: Evolution of Cannibalistic Traits: Scenarios Derived from Adaptive Dynamics. IIASA Interim Report IR-02-054 (2002).

Issues of the IIASA Studies in Adaptive Dynamics series can be obtained at www.iiasa.ac.at/Research/ADN/Series.html or by writing to adn@iiasa.ac.at. 


\section{Contents}

1 Introduction 1

2 The resident-mutant model 2

3 Monomorphic dynamics $\quad 4$

4 Dimorphic dynamics 6

5 Discussion and conclusions $\quad 10$

6 Appendix: Analysis of the monomorphic canonical equation 13 


\begin{abstract}
The evolution of cannibalistic traits in consumer populations is studied in this paper with the approach of Adaptive Dynamics theory. The model is kept at its minimum complexity by eliminating some environmental characteristics, like heterogeneity and seasonalities, and by hiding the sizestructure of the population. Evolutionary dynamics are identified through numerical bifurcation analysis, applied both to the ecological (resident-mutant) model and to the canonical equation of Adaptive Dynamics. The result is a rich catalogue of evolutionary scenarios involving evolutionary stable strategies and branching points both in the monomorphic and dimorphic dynamics. The possibility of evolutionary extinction of highly cannibalistic populations is also ascertained. This allows one to explain why cannibalism can be a transient stage of evolution.
\end{abstract}




\author{
About the Authors \\ Fabio Dercole \\ CIRITA, Politecnico di Milano \\ Via Ponzio 34/5, 20133 Milano, Italy \\ A-2361 Laxenburg, Austria \\ Sergio Rinaldi \\ CIRITA, Politecnico di Milano \\ Via Ponzio 34/5, 20133 Milano, Italy \\ and \\ Adaptive Dynamics Network \\ International Institute for Applied Systems Analysis \\ A-2361 Laxenburg, Austria
}

\title{
Acknowledgement
}

The authors are grateful to David Claessen for his help during the first stage of this study, to Hans Metz for his encouragement and useful suggestions, and to Ulf Dieckmann who invited them to participate to the Adaptive Dynamics Network program at the International Institute for Applied Systems Analysis, Laxenburg, Austria. The support of the European Science Foundation to F. D. and of CESTIA (CNR, Milano, Italy) to S. R. is also acknowledged. 


\title{
Evolution of Cannibalistic Traits: Scenarios Derived from Adaptive Dynamics
}

\author{
Fabio Dercole \\ Sergio Rinaldi
}

\section{Introduction}

Cannibalism, defined as intraspecific predation, is a behavioral trait found in a wide variety of animals, ranging from protozoa and rotifers to birds and mammals (Fox, 1975). The most important studies based on field and laboratory data have been surveyed by Polis $(1981,1988)$ who has shown that pronounced cannibalism is a frequent feature of population dynamics in species that grow through a wide size range. Often cannibalism develops at ecological time scale as a reaction of adult individuals to food scarcity (Fox, 1975). However, besides the evidence for dietary induction several types of data indicate that, for many species, there is a strong genetic component to cannibalism (see Polis (1981) and references therein).

The aim of this note is to show how a few characteristics of the evolution of phenotypic cannibalistic traits in consumer populations can be derived from general and formal principles. The approach we follow is that of Adaptive Dynamics Theory (Hofbauer and Sigmund, 1990; Metz et al., 1992; Geritz et al., 1997). It is based on a transparent conceptual framework (small and rare random mutations followed by natural selection) and allows one to describe the dynamics of the traits in a purely deterministic way, through an ODE called canonical equation (Dieckmann and Law, 1996; Champagnat et al., 2001). The method is also capable of explaining the transition from monomorphism to dimorphism (Geritz et al., 1997).

However, the derivation of the canonical equation poses some problems if the resident and mutant populations are described with high dimensional models. On the other hand, cannibalistic consumer populations naturally call for relatively complex age/size-structured models (Polis, 1988). Thus, in order to easily derive the canonical equation we have used a strongly simplified population model. Our choice has been to hide the size-structure of the population as well as all environmental heterogeneity and seasonalities, which are known to enhance cannibalism in many species (Fox, 1975). Thus, both the resident and the mutant populations are described with a first order ODE with constant parameters. Although the model on which the entire study is based is only a caricature of the real world, it contains the basic ingredients for a sound discussion of adaptation. In fact, the cannibalistic predation rate and the searching efficiency of the common resource depend upon a phenotypic trait from now on called cannibalism. Moreover, the functional form of the model and the ranges of its admissible parameter values have been carefully selected in order to fit a paradigmatic case, namely that of the Eurasian perch (Perca fluviatilis), recently described in great detail (Claessen et al., 2000). Thus, at least from this point of view, the model is quite realistic.

The paper is organized as follows. In the next section we describe the resident-mutant model and the dependence of the demographic parameters upon the adaptive trait. In the third section we derive the monomorphic canonical equation and study the evolutionary dynamics of the trait. In particular, we show that an evolutionary stable strategy (ESS) (Maynard Smith and Price, 1973; Maynard Smith, 1982) characterized by a low value of cannibalism is always guaranteed if the 
environment is not too rich, and that dimorphism is a possible evolutionary option in populations with wide size range. Then, in the following section we explore dimorphic dynamics by studying a second order canonical equation derived, once more, from the resident-mutant model. The most interesting result is that dimorphic evolution can have a halt in an ESS characterized by the coexistence of two populations, one with low and one with high cannibalism. Assuming that body size of adult individuals and cannibalism are positively correlated, this dimorphic ESS explains the coexistence of $d w a r f s$ and giants. Our findings are therefore consistent with one of the conclusions of Polis (1988) who assessed "the possible evolutionary options of large entities living among hordes of smaller entities".

\section{The resident-mutant model}

Assume that a cannibalistic consumer population is characterized by a phenotypic trait indicated by $x$. Since we do not want to refer to a particular population or species, we can not specify what $x$ is. However, in order to facilitate the interpretation of our results, we take the liberty of assuming that the size of adult individuals is positively correlated with the cannibalistic trait. Thus, $x$ can be simply identified with a suitable measure of body size, so that the coexistence of two subpopulations, one with low and one with high cannibalism, should be revealed by the presence of dwarfs and giants in the same environment.

The derivation of the canonical equation of Adaptive Dynamics requires two things: $(i)$ the knowledge of the interactions occurring at ecological time scale between all sub-populations; $(i i)$ the dependence of the demographic parameters of the sub-populations upon the traits. All this can be specified through a resident-mutant model composed of $(N+1)$ populations. The first $N$ populations, with biomass densities $n_{i}$ and traits $x_{i}$, are the resident populations, while the last population, with biomass density $n_{N+1}$ and trait $x_{N+1}$, is the mutant population. The interactions between all sub-populations are described by the following ODE's

$$
\dot{n}_{i}=n_{i}\left(\frac{\sum_{j=0}^{N+1} e_{i j} a_{i j} n_{j}}{1+\sum_{j=0}^{N+1} h_{i j} a_{i j} n_{j}}-\sum_{j=1}^{N+1} \frac{a_{j i} n_{j}}{1+\sum_{k=0}^{N+1} h_{j k} a_{j k} n_{k}}-\sum_{j=1}^{N+1} c_{i j} n_{j}\right) \quad i=1, \ldots, N+1
$$

where the index 0 refers to the common resource and the indexes $1, \ldots, N+1$ to the consumer subpopulations. Notice that the density $n_{0}$ of the common resource, from now on called environmental richness, is assumed to be constant, i. e. seasonalities are ruled out. The three terms at the righthand-side of eq. (1) are natality due to food intake, mortality due to cannibalism and mortality due to competition. The first term is written in the form of a type II functional response and takes into account that each individual has two alternative food sources: the common resource and the individuals of the same species. In the case of the Eurasian perch, which has motivated the present study, the common resource is zooplankton on which all perch feed, at least in the first stages of their life (Holcik, 1977). Thus, rich environment are those in which young perch have more access to food. The parameter $e_{i j}$ is a conversion factor transforming food intake of type $j$ into new biomass of type $i$. The parameter $h_{i j}$ is the handling time of the $i$-th sub-population associated with the food source of type $j$ and $c_{i j}$ is a coefficient specifying the extra-mortality due to competition. Although all demographic parameters depend upon various traits, in order to obtain a tractable problem we limit the analysis to the case in which only two parameters depend upon the trait we have called cannibalism. Our choice has been to assume that the parameters $e_{i j}$ and $c_{i j}$ are constant (recall that $n_{i}$ is biomass density), while the attack rates $a_{i j}$ and the handling times $h_{i j}$ depend upon the traits. But other choices would also be justifiable. 


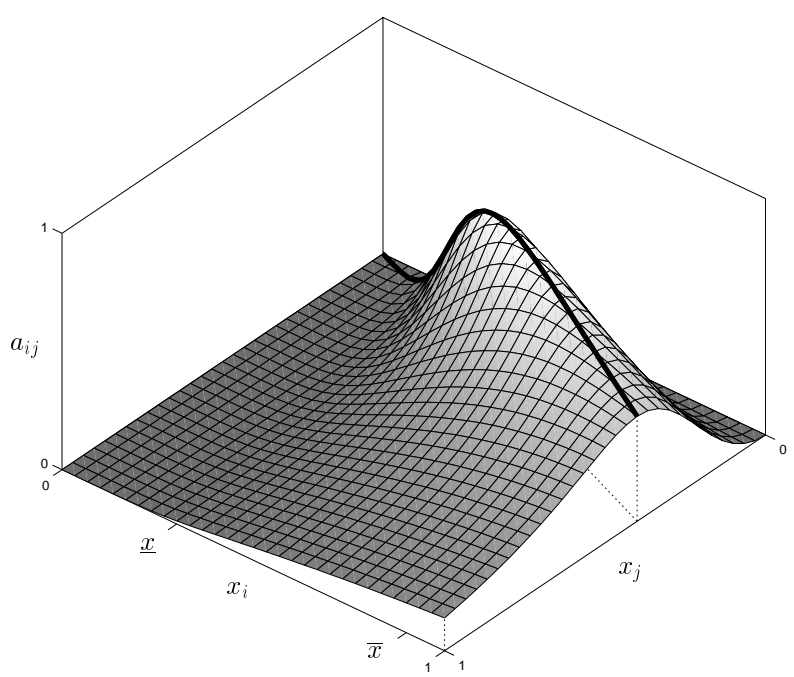

Figure 1: The cannibalistic attack rate $a_{i j}$ as a function of the traits $x_{i}$ and $x_{j}$ (see eq. (3)). The thick line indicates the restriction of $a_{i j}$ on the ray $x_{j}=p x_{i}$. Parameter values: $A_{i j}=1, \beta=2, \gamma=4, \delta=2$, $\underline{x}=0.3, \bar{x}=0.9, p=0.4$.

The attack rate $a_{i 0}$ specifies the consumption of the common resource and is assumed to be a bell-shaped function of the trait $x_{i}$, because a consumer performs better when its body size is well tuned with the size of the local resource. The trait value at which the attack rate is maximum is supposed to be the same for all sub-populations and is indicated by $x^{0}$. In the analysis we use the following bell-shaped function

$$
a_{i 0}=\frac{2 A_{i 0}}{\left(\frac{x_{i}}{x^{0}}\right)^{\alpha}+\left(\frac{x^{0}}{x_{i}}\right)^{\alpha}}
$$

where $A_{i 0}$ is the maximum attack rate and $\alpha>1$ specifies the sharpness of the bell.

As for the cannibalistic attack rate $a_{i j}$, we assume it is shaped as in Figure 1. Along each ray $x_{j} / x_{i}=$ const the attack rate is bell-shaped and vanishes for $x_{i}$ tending to zero and to infinity. Similarly, $a_{i j}$ is a bell-shaped function of the ratio $x_{j} / x_{i}$, since the predation rate is higher when the body size of the victim is in a suitable ratio with that of the predator, i. e. when $x_{j}=p x_{i}$, $p<1$. The function we use in our analysis is

$$
a_{i j}=A_{i j}\left[\frac{2}{\left(\frac{p x_{i}}{x_{j}}\right)^{\beta}+\left(\frac{x_{j}}{p x_{i}}\right)^{\beta}}\right]\left(\frac{x_{i}^{\gamma}}{\underline{x}^{\gamma}+x_{i}^{\gamma}}\right)\left(1-\frac{x_{i}^{\delta}}{\bar{x}^{\delta}+x_{i}^{\delta}}\right)
$$

where $A_{i j}$ is the maximum attack rate and $\beta>1, \gamma>1, \delta>1$ and $\underline{x}, \bar{x}$ are suitable parameters specifying the bell-shaped functions. The parameter $\underline{x}$ is a sort of threshold indicating the body size at which cannibalism becomes physiologically significant, while the second threshold $\bar{x}$ is the body size at which predation starts to be limited by habitat morphology (see Figure 1). In order to allow the survival of populations with negligible cannibalism $\left(x_{i}<\underline{x}\right)$ we assume in the following $x^{0}<\underline{x}$. Small values of $\beta$ imply high values of the cannibalistic attack rate $a_{i i}$ (see eq. (3) with $x_{i}=x_{j}$ ), i. e. great possibilities for individuals of trait $x_{i}$ to predate individuals of the same trait. In the real world such a population would be characterized by a substantial change in size from juvenile to adult, so that adult individuals can easily predate young ones (Polis, 1981, 1988). For this reason the parameter $(1 / \beta)$ is a sort of surrogate for the size range of the individuals in the population and will, indeed, be called size range in the following. 
Finally, the handling times $h_{i j}$, which can be estimated from feeding experiments performed under excessive food conditions (Byström and Garcia-Berthóu, 1999), are assumed to depend mainly upon the trait $x_{i}$ through the function (see Claessen et al., 2000)

$$
h_{i j}=w_{1} x_{i}^{-w_{2}}
$$

For this reason, in the Appendix the functions $h_{i j}$ are substituted by $h_{i}$.

\section{Monomorphic dynamics}

We now use model (1-4) with $N=1$ to study the monomorphic evolution of cannibalism. Consistently with the Adaptive Dynamics approach we assume that the resident population with trait value $x_{1}$ is at its equilibrium $\bar{n}_{1}\left(x_{1}\right)$ when a mutant appears. The uniqueness of this equilibrium can be easily ascertained from the formulas presented in the Appendix. Moreover, we also assume that the trait $x_{2}$ of the mutant is only slightly different from $x_{1}$ (i. e. $x_{2}=x_{1}+\epsilon$, with small $\epsilon$ ) and that the mutant population density $n_{2}$ is initially very small. Under these conditions, model (1-4) written in the form

$$
\begin{aligned}
& \dot{n}_{1}=n_{1} f_{1}\left(n_{1}, n_{2}, x_{1}, x_{2}\right) \\
& \dot{n}_{2}=n_{2} f_{2}\left(n_{1}, n_{2}, x_{1}, x_{2}\right)
\end{aligned}
$$

can be used to establish the fate of the mutant and resident populations. Generically, an invading mutant replaces the former resident so that, in the end, the system is composed of a single population with trait $x_{1}+\epsilon$. In the opposite case, i. e. when the mutant population does not invade, it goes extinct so that the trait of the population remains unchanged. This process of mutation and selection can be further specified by making suitable assumptions on the frequency and distribution of small mutations (Dieckmann and Law, 1996) and the conclusion is that the rate at which the trait $x_{1}$ varies at evolutionary time scale is given by the following ODE (called canonical equation of Adaptive Dynamics)

$$
\dot{x}_{1}=\left.k \bar{n}_{1}\left(x_{1}\right) \frac{\partial \bar{f}_{2}\left(x_{1}, x_{2}\right)}{\partial x_{2}}\right|_{x_{2}=x_{1}}
$$

where $k$ is proportional to the frequency and variance of small mutations and $\bar{f}_{2}\left(x_{1}, x_{2}\right)$ is the fitness of the mutant, i. e.

$$
\bar{f}_{2}\left(x_{1}, x_{2}\right)=f_{2}\left(\bar{n}_{1}\left(x_{1}\right), 0, x_{1}, x_{2}\right)
$$

Equation (6) always admits the trivial solution $x_{1}=0$ because $\bar{n}_{1}\left(x_{1}\right)$ and $\partial \bar{f}_{2} /\left.\partial x_{2}\right|_{x_{2}=x_{1}}$ are zero for $x_{1}=0$ (the proof can be easily derived from eqs. (A1,A3) of the Appendix, by taking into account that $a_{10}$ vanishes for $x_{1}$ tending to zero (see eq. (2))). Moreover, the trivial solution $x_{1}=0$ is always unstable (i. e. $\dot{x}_{1}>0$ for small $x_{1}>0$ ) since $\bar{n}_{1}\left(x_{1}\right)$ and $\partial \bar{f}_{2} /\left.\partial x_{2}\right|_{x_{2}=x_{1}}$ are positive for small and positive values of $x_{1}$. Since $k>0$ and $\bar{n}_{1}\left(x_{1}\right)$ is positive for any positive $x_{1}$, eqs. $(6,7)$ say that $x_{1}$ is stationary (monomorphic equilibrium) when the fitness of the mutant is stationary with respect to $x_{2}$. In generic conditions, the non-trivial monomorphic equilibria are either one or three, as shown in Figure 2 for three different combinations of environmental richness $\left(n_{0}\right)$ and size range $(1 / \beta)$ (see the Appendix for a qualitative analysis of eqs. $\left.(6,7)\right)$. In the case of Figure 2B two stable equilibria $\bar{x}_{1}^{\prime}$ and $\bar{x}_{1}^{\prime \prime \prime}$ (filled circles on the $x_{1}$ axis) are separated by an unstable equilibrium $\bar{x}_{1}^{\prime \prime}$ (empty circle). Thus, in this case the cannibalistic trait can evolve either toward a low value (corresponding to a very dense population of dwarfs) or toward a high value (corresponding to a scarce population of giants). In the other two cases there is only one stable equilibrium: a low value $\bar{x}_{1}^{\prime}$ with high population density in case $\mathrm{A}$, and a high value $\bar{x}_{1}^{\prime \prime \prime}$ with low population density in case $\mathrm{C}$. The transition from $\mathrm{B}$ to $\mathrm{A}[\mathrm{C}]$ is characterized by the collision of $\bar{x}_{1}^{\prime \prime}$ with $\bar{x}_{1}^{\prime \prime \prime}\left[\bar{x}_{1}^{\prime}\right]$. The parameter conditions characterizing such collisions can, in principle, be detected through extensive simulations of model $(6,7)$. However, they can be detected much 


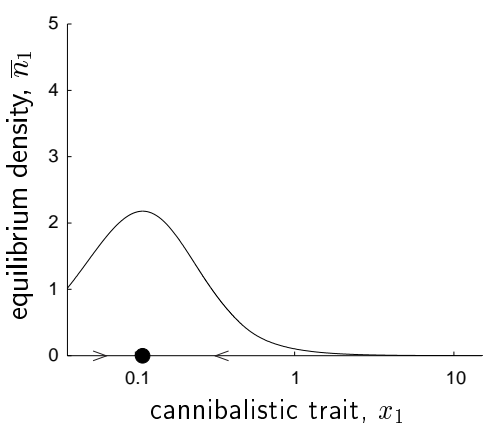

(A)

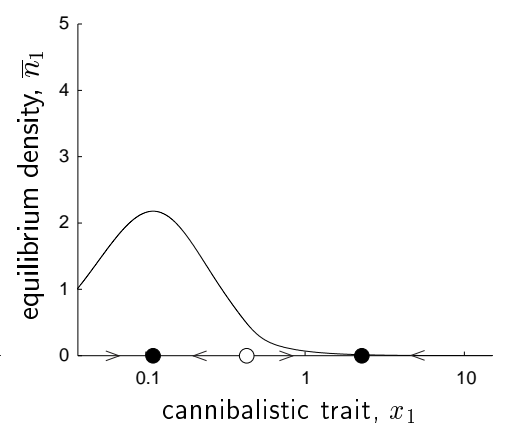

(B)

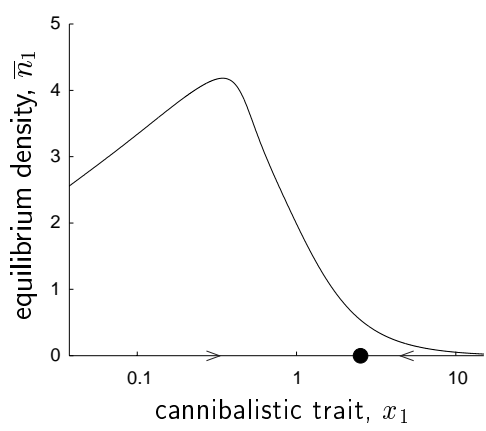

$(C)$

Figure 2: The equilibrium density $\bar{n}_{1}\left(x_{1}\right)$ of model (1-4) and monomorphic dynamics on the horizontal axis, where circles indicate equilibria of model $(6,7)$. $(A) n_{0}=10, \beta=2.5$. $(B) n_{0}=10, \beta=1.5$. $(C)$ $n_{0}=500, \beta=1.5$. Other parameter values: $A_{i 0}=1, A_{i j}=10, x^{0}=0.1, \underline{x}=0.5, \bar{x}=5, p=0.2$, $\alpha=2, \gamma=8, \delta=2, c=1, e=0.6, w_{1}=0.1, w_{2}=0.25, \mathrm{i}=1,2$.

more accurately through numeric bifurcation analysis (Kuznetsov, 1998), which is, indeed, the technique used in this paper.

Once monomorphic dynamics have found a halt at a stable monomorphic equilibrium $\left(\bar{x}_{1}^{\prime}\right.$ or $\bar{x}_{1}^{\prime \prime \prime}$ in our case), one should look at the higher order terms in the Taylor expansion of the fitness function (7) to establish if the equilibrium is an ESS or a branching point (Geritz et al., 1997). More precisely, at a stable monomorphic equilibrium, the following correspondence holds

$$
\begin{array}{ll}
\left.\frac{\partial^{2} \bar{f}_{2}\left(x_{1}, x_{2}\right)}{\partial x_{2}^{2}}\right|_{x_{2}=x_{1}}<0 & \text { ESS } \\
\left.\frac{\partial^{2} \bar{f}_{2}\left(x_{1}, x_{2}\right)}{\partial x_{2}^{2}}\right|_{x_{2}=x_{1}}>0 & \text { branching point }
\end{array}
$$

In the first case all small mutations of the resident population fail to invade, while in the second case small mutations invade but do not replace the former resident. Thus, branching points are the origin of dimorphism. For example, in Figure 2 the low equilibria $\bar{x}_{1}^{\prime}$ are ESSs, while the high equilibria $\bar{x}_{1}^{\prime \prime \prime}$ are branching points (see Appendix). But other combinations are possible for other values of environmental richness $\left(n_{0}\right)$ and size range $(1 / \beta)$.

The study of monomorphic dynamics has been completed by performing the bifurcation analysis of model $(6,7)$ with respect to $\left(n_{0}\right)$ and $(1 / \beta)$, thus producing the diagram shown in Figure 3. In such a diagram, the two curves merging at the cusp point $C$ are the combinations of parameter values $\left(n_{0}, 1 / \beta\right)$ for which the unstable equilibrium $\bar{x}_{1}^{\prime \prime}$ collides with $\bar{x}_{1}^{\prime}$ or $\bar{x}_{1}^{\prime \prime \prime}$. By contrast, the remaining curve represents the values $\left(n_{0}, 1 / \beta\right)$ for which $\partial^{2} \bar{f}_{2} /\left.\partial x_{2}^{2}\right|_{x_{2}=x_{1}}$ evaluated at $\bar{x}_{1}^{\prime \prime \prime}$ is zero, i. e. the values $\left(n_{0}, 1 / \beta\right)$ separating evolutionary stable strategies from branching strategies (see eq. (8)). Thus, the space $\left(n_{0}, 1 / \beta\right)$ is subdivided into four regions, each characterized by one or two stable monomorphic equilibria and by a different mix of ESSs and branching points. In particular, Figure 3 shows that in poor environments an ESS always exists and that dimorphism (due to branching points) is a possible evolutionary option only in populations with wide size range (actually it is the only option in very rich environments). Through Figure 3 one can also identify the conditions under which a population will evolve toward high degrees of cannibalism. Indeed, in the regions with wide size range the presence of a branching point and the fact that its associated cannibalistic trait is high (see point $\bar{x}_{1}^{\prime \prime \prime}$ in Figure $2 \mathrm{~B}$ ) guarantees the possibility of a monomorphic evolution toward high degrees of cannibalism (followed by a subsequent phase of dimorphism discussed in the next section). However, populations characterized by small size 


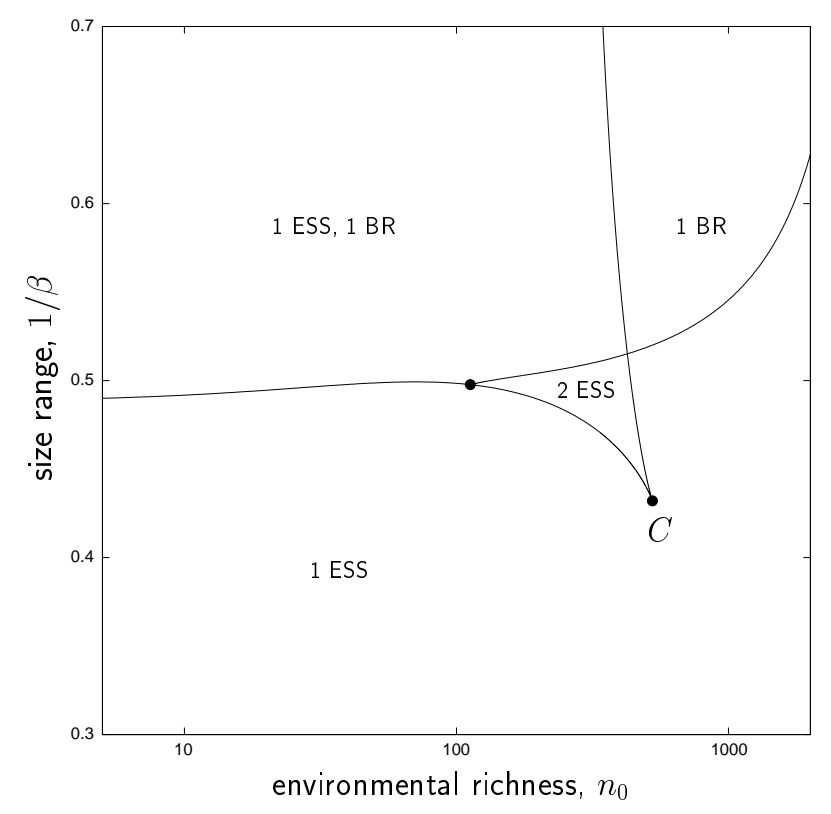

Figure 3: Bifurcation diagram of model $(6,7)$ with respect to $n_{0}$ and $1 / \beta$. The curves identify four regions characterized by one or two stable monomorphic equilibria which can be either evolutionary stable strategies (ESS) or branching points (BR). Parameter values as in Figure 2.

ranges can also develop high degrees of cannibalism, because it can be shown through numerical analysis that the cannibalistic trait associated to the ESS is high for very rich environments. All this is in good agreement with Polis $(1981,1988)$, who has noticed that pronounced cannibalism is often present in populations growing through a wide size range.

\section{Dimorphic dynamics}

We now focus on the evolution of the cannibalistic traits $x_{1}$ and $x_{2}$ of two coexisting sub-populations with densities $n_{1}$ and $n_{2}$. The aim of the analysis is twofold. First we want to investigate the long term evolution of the traits and establish, in particular, if dimorphism is the final state of evolution or can turn into polymorphism or even back to monomorphism (Matsuda and Abrams, 1994a,b; Dieckmann et al., 1995). Second, we want to show that a sort of catalogue of all possible outcomes can be identified by performing, once more, a bifurcation analysis with respect to parameters.

The study of dimorphic dynamics must be limited to the coexistence region, which is the region of all pairs $\left(x_{1}, x_{2}\right)$ for which model (5) has a stable and strictly positive equilibrium. Such a region can be computed by performing the bifurcation analysis of model (5) with respect to the traits $x_{1}$ and $x_{2}$ interpreted as constant parameters. Since dimorphic dynamics, i. e. trajectories in the space $\left(x_{1}, x_{2}\right)$, are symmetric with respect to the diagonal $x_{2}=x_{1}$, we limit the analysis to the region $x_{1}<x_{2}$ and call populations 1 and 2 dwarf and giant populations, respectively. An example of this bifurcation analysis is shown in Figure 4, where the upper part reports all bifurcation curves which identify seven regions (I-VII), while the lower part reports the corresponding state portraits of model (5). Since only in the state portraits IV and VII there is a stable and strictly positive equilibrium, the region of coexistence is the union of regions IV and VII. The points $E$, $U$ and $B$ on the diagonal $x_{2}=x_{1}$, where various bifurcation curves merge, correspond to the monomorphic equilibria, i. e. $E \equiv\left(\bar{x}_{1}^{\prime}, \bar{x}_{1}^{\prime}\right), U \equiv\left(\bar{x}_{1}^{\prime \prime}, \bar{x}_{1}^{\prime \prime}\right)$, and $B \equiv\left(\bar{x}_{1}^{\prime \prime \prime}, \bar{x}_{1}^{\prime \prime \prime}\right)$. Since Figure 4 has been obtained for the same parameter settings used in Figure $2 \mathrm{~B}$, the equilibria $\bar{x}_{1}^{\prime}, \bar{x}_{1}^{\prime \prime}$, and 


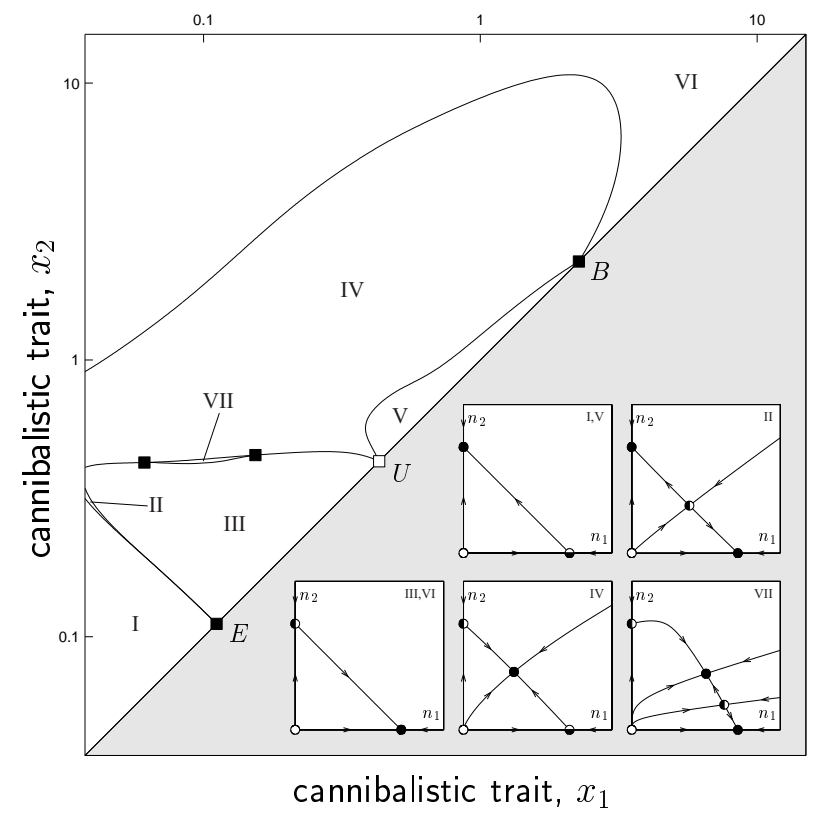

Figure 4: Bifurcation diagram of model (5) with respect to cannibalistic traits $x_{1}$ and $x_{2}$. Parameter values as in Figure 2B. Upper triangle: bifurcation curves and regions I-VII (squares indicate codimension2 bifurcation points). Lower triangle: state portraits of model (5) for each region I-VII (circles indicate equilibria of model (5)).

$\bar{x}_{1}^{\prime \prime \prime}$ of model $(6,7)$ are ESS, unstable, and branching, respectively. The nature of a bifurcation curve separating two nearby regions can be understood by comparing the two corresponding state portraits. For example, the bifurcation curve separating region IV from region VI is characterized (see state portraits IV and VI) by the collision of a stable node with a saddle on the $n_{1}$-axis. Thus, if a dimorphic trajectory in region IV moves toward and finally hits this bifurcation curve, the giant population goes extinct. In such a case the bifurcation curve corresponds to what is properly called evolutionary murder. In fact, approaching this curve, $\dot{x}_{2}$ vanishes (see forthcoming eq. (10)), i. e. it is the evolutionary change in the dwarfs that kills the giants, thus marking the transition from dimorphism to monomorphism.

We now use model (1-4) with $N=2$ and denote by $\bar{n}_{1}\left(x_{1}, x_{2}\right)$ and $\bar{n}_{2}\left(x_{1}, x_{2}\right)$ the densities of the stable and strictly positive equilibrium of model (5) in the region of coexistence. As for monomorphic evolution, we assume that the resident populations are at equilibrium when a mutant appears. Moreover, the mutant population is initially very scarce and its trait $x_{3}$ is only slightly different from that of the resident population (i. e. $x_{3}=x_{i}+\epsilon$, with small $\epsilon$ and $i=1$ or 2 ). If model (1-4) is written in the form

$$
\begin{aligned}
& \dot{n}_{1}=n_{1} f_{1}\left(n_{1}, n_{2}, n_{3}, x_{1}, x_{2}, x_{3}\right) \\
& \dot{n}_{2}=n_{2} f_{2}\left(n_{1}, n_{2}, n_{3}, x_{1}, x_{2}, x_{3}\right) \\
& \dot{n}_{3}=n_{3} f_{3}\left(n_{1}, n_{2}, n_{3}, x_{1}, x_{2}, x_{3}\right)
\end{aligned}
$$

the dimorphic canonical equation turns out to be given by

$$
\begin{aligned}
& \dot{x}_{1}=\left.k_{1} \bar{n}_{1}\left(x_{1}, x_{2}\right) \frac{\partial \bar{f}_{3}\left(x_{1}, x_{2}, x_{3}\right)}{\partial x_{3}}\right|_{x_{3}=x_{1}} \\
& \dot{x}_{2}=\left.k_{2} \bar{n}_{2}\left(x_{1}, x_{2}\right) \frac{\partial \bar{f}_{3}\left(x_{1}, x_{2}, x_{3}\right)}{\partial x_{3}}\right|_{x_{3}=x_{2}}
\end{aligned}
$$

where $k_{1}$ and $k_{2}$ are proportional to the frequency and variance of small mutations in the resident 
populations and $\bar{f}_{3}\left(x_{1}, x_{2}, x_{3}\right)$ is the fitness of the mutant, i. e.

$$
\bar{f}_{3}\left(x_{1}, x_{2}, x_{3}\right)=f_{3}\left(\bar{n}_{1}\left(x_{1}, x_{2}\right), \bar{n}_{2}\left(x_{1}, x_{2}\right), 0, x_{1}, x_{2}, x_{3}\right)
$$

Moreover, if dimorphic dynamics find a halt at a stable equilibrium $\left(\bar{x}_{1}, \bar{x}_{2}\right)$, such an equilibrium is an ESS if

$$
\left.\frac{\partial^{2} \bar{f}_{3}\left(\bar{x}_{1}, \bar{x}_{2}, x_{3}\right)}{\partial x_{3}^{2}}\right|_{x_{3}=\bar{x}_{i}}<0 \quad i=1,2
$$

Conversely, if condition (12) does not hold, then the dimorphic equilibrium is a branching point.

Three examples of dimorphic dynamics are shown in Figure 5 for different values of environmental richness $\left(n_{0}\right)$ and size range $(1 / \beta)$. The coexistence region is partitioned in white and dark subregions. Trajectories starting in the white region tend toward a dimorphic equilibrium $D$ which can be either ESS or branching. By contrast, trajectories starting in the dark region hit the boundary of the coexistence region where an evolutionary murder occurs.

In Figure 5A, the trajectories starting close to the branching point $B$ where dimorphism originates, tend toward a dimorphic stable ESS $D \equiv\left(\bar{x}_{1}, \bar{x}_{2}\right)$, characterized by $\bar{n}_{1}\left(\bar{x}_{1}, \bar{x}_{2}\right)>$ $\bar{n}_{2}\left(\bar{x}_{1}, \bar{x}_{2}\right)$. This result is in agreement with Polis (1988) observation on the possible coexistence of an abundant population of small individuals with a scarce population of large and highly cannibalistic individuals.

Figure 5B corresponds to populations with a wider size range $(1 / \beta)$. The cannibalism of the monomorphic population at the branching point is so high that point $B$ is now on the boundary of the dark region, so that dimorphic dynamics end with the evolutionary murder of the giant population at point $X^{*} \equiv\left(x_{1}^{*}, x_{2}^{*}\right)$. After that (i. e. after the sudden transition from $X^{*}$ to $X^{* *}$ in Figure 5B) the dwarf population evolves, starting with a trait $x_{1}=x_{1}^{*}$ in accordance with the monomorphic canonical equations $(6,7)$. Thus, in the end, the system settles at the monomorphic ESS $\bar{x}_{1}^{\prime}$ (see point $E$ of Figure 5B). In other words, starting from any ancestral monomorphic condition the final outcome of evolution is a low cannibalistic population of dwarfs. However, if the ancestral conditions are characterized by a sufficiently low cannibalistic trait (i. e. $x_{1}<$ $\bar{x}_{1}^{\prime \prime}$, see point $U$ in Figure 5B), the evolution is purely monomorphic, while ancestral conditions $x_{1}>\bar{x}_{1}^{\prime \prime}$ give rise to three distinct evolutionary phases: first a monomorphic evolution toward the branching point $B$, then a dimorphic evolution implying the temporary presence of a highly cannibalistic population of giants (from $B$ to $X^{*}$ ) and, finally, after the extinction of the giant population, a monomorphic evolution toward an ESS (from $X^{* *}$ to $E$ ). All this can be summarized by saying that in evolutionary systems different but very close initial conditions can generate completely different evolutionary paths, ending however in the same final state (see also Geritz et al., 1999). This property (which does not hold in generic dynamical systems) might be crucial for understanding controversial results based on field or laboratory data concerning the evolution of adaptive traits.

In the case of a richer environment (see Figure 5C) the trajectories starting close to the branching point $B$ tend toward a stable dimorphic equilibrium $D \equiv\left(\bar{x}_{1}, \bar{x}_{2}\right)$, as in the case of Figure 5A. However, at point $D$ condition (12) holds only for $i=1$, so that at $D$ the giant population undergoes a branching. Of course, the procedure we have followed to construct the monomorphic and dimorphic canonical equations can be extended to the general polymorphic case. In particular, for the parameter values of Figure 5C, numerical simulations show that the trajectory of the polymorphic canonical equation with $N=3$ starting from $\left(\bar{x}_{1}, \bar{x}_{2}, \bar{x}_{2}+\epsilon\right)$, tends toward a polymorphic stable ESS characterized by a scarce population of giants, a crowded population of dwarfs and a population of individuals with intermediate body size. Also this result is not in conflict with the observations described in Polis (1988).

The complete bifurcation analysis of model $(10,11)$ is out from the scope of this paper. However, by looking at Figure 5 we can make the following considerations. The transition between 

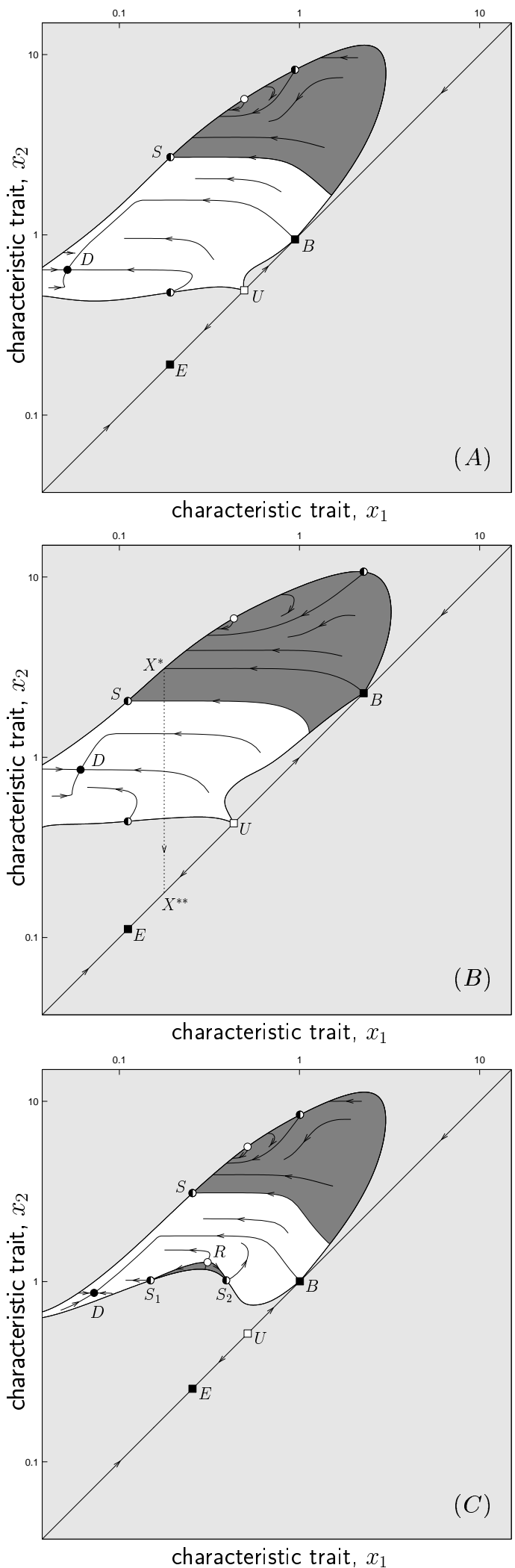

Figure 5: Examples of dimorphic dynamics. $(A) n_{0}=10, \beta=1.9$. (B) $n_{0}=10, \beta=1.5 .(C) n_{0}=50$, $\beta=1.9$ (a small part of the coexistence region attached to point $U$ and not connected to the rest of the coexistence region is not shown). Other parameter values as in Figure 2. 
the state portraits of Figures 5A and B is a global bifurcation (called heteroclinic bifurcation) in which the unstable manifold of point $B$ coincides with the stable manifold of the saddle $S$, while the transition between the state portraits of Figures $5 \mathrm{~A}$ and $\mathrm{C}$ is slightly more complicated. In fact, two characteristics of the state portrait of Figure 5C are qualitatively different from those of Figure 5A: first point $U$ does not belong to the coexistence region, and second there is a small dark region in which the dimorphic dynamics are characterized by the evolutionary murder of the dwarf population. Starting from Figure 5A and increasing $n_{0}$, at a first critical value $n_{0}^{\prime}$ the coexistence region looses the contact with point $U$. This implies that, for $n_{0}$ slightly bigger than $n_{0}^{\prime}$, the border of the coexistence region on the left of point $B$ is characterized by the absence of the dwarf population. This first bifurcation can be identified by a straightforward condition on the fitness (7). At a second critical value $n_{0}^{\prime \prime}$ the equilibria $R, S_{1}$ and $S_{2}$ appear contemporarily at a single point of the border of the coexistence region. For a further increase of $n_{0}$ the three points split: the repellor $R$ enters into the coexistence region, while the saddles $S_{1}$ and $S_{2}$ remain on its border, thus giving rise to the small dark region of Figure $5 \mathrm{C}$. The bifurcation at $n_{0}=n_{0}^{\prime \prime}$, called pitchfork bifurcation, can be easily identified with the condition $\bar{n}_{1}\left(x_{1}^{R}, x_{2}^{R}\right)=0$, where $\left(x_{1}^{R}, x_{2}^{R}\right)$ are the coordinates of $R$. Finally, at a third critical value $n_{0}^{\prime \prime \prime}$ the dimorphic ESS $D$ becomes a branching point. Strictly speaking, this critical value is not a bifurcation of model $(10,11)$. However, it implies a discontinuity in the evolutionary dynamics, i. e. the birth of polymorphism with $N=3$, so that it is justified to consider it as a special bifurcation.

All the bifurcations described above and others not involved in the transitions between the state portraits of Figure 5 can be continued in a two parameter space, e. g. $\left(n_{0}, 1 / \beta\right)$. However, this poses nontrivial technical problems. In fact, the continuation of global bifurcations requires to solve specific boundary-value problems for model $(10,11)$ in which $\bar{n}_{1}\left(x_{1}, x_{2}\right)$ and $\bar{n}_{2}\left(x_{1}, x_{2}\right)$ are not known in closed form. Thus, the bifurcation analysis of model $(10,11)$ must be performed by considering differential algebraic systems of the form

$$
\begin{aligned}
\dot{x}_{1} & =\left.k_{1} n_{1} \frac{\partial f_{3}\left(n_{1}, n_{2}, 0, x_{1}, x_{2}, x_{3}\right)}{\partial x_{3}}\right|_{x_{3}=x_{1}} \\
\dot{x}_{2} & =\left.k_{2} n_{2} \frac{\partial f_{3}\left(n_{1}, n_{2}, 0, x_{1}, x_{2}, x_{3}\right)}{\partial x_{3}}\right|_{x_{3}=x_{2}} \\
0 & =f_{1}\left(n_{1}, n_{2}, 0, x_{1}, x_{2}, 0\right) \\
0 & =f_{2}\left(n_{1}, n_{2}, 0, x_{1}, x_{2}, 0\right)
\end{aligned}
$$

for which algorithms for the numerical solution of boundary-value problems are hard to develop (see, however, Ascher and Spiteri (1994)).

\section{Discussion and conclusions}

The problem of evolution of cannibalistic traits in consumer populations has been investigated in this paper. The approach has been purely abstract (Adaptive Dynamics theory) and based on a very simple model. Important environmental features like heterogeneity of the habitat and seasonalities, have not been taken into account, while a great deal of attention has been given to 'environmental richness'. In order to keep the model at the minimum degree of complexity, we have also hidden the size-structure of the population, which has been, however, indirectly taken into account through a specific parameter called 'size range'.

The study has been performed through extensive bifurcation analysis of both the ecological model and the evolutionary model. The result is a rich catalogue of possible evolutionary scenarios. In poor habitats, population with small size range remain monomorphic and tend to an ESS characterized by a dense population of dwarfs in which cannibalism is practically absent. The characteristics of the monomorphic ESS change smoothly with the richness of the environment 
until for very rich environments the ESS is characterized by a scarce population of giants, namely a population of large and highly cannibalistic adult individuals. By contrast, monomorphic populations with wide size range can converge to a branching point which is the starting point of a dimorphic phase, which depending upon the cases, can be of various form. First of all, we can have convergence to a dimorphic ESS, characterized by a dense population of dwarfs and a scarce population of giants. In this case, dimorphism is the final state of evolution. But dimorphism can be also a transient stage of evolution. This happens when dimorphic dynamics converge to a branching point, from which a new mutant population invades, thus giving rise to a higher order polymorphism. Surprisingly, also the opposite transition (from dimorphism to monomorphism) can occur through the evolutionary murder of the giant population. In other words, for suitable demographic and environmental conditions we can have a rather interesting evolutionary path: a monomorphic population first increases its degree of cannibalism thus becoming a population of giants when approaching a branching point; then, after branching, the giant population becomes more and more scarce (at evolutionary time scale) until it goes extinct; finally, the remaining monomorphic population settles at an ESS characterized by a huge number of dwarfs. In conclusion, our analysis shows that depending on the ancestral conditions and on the demographic and environmental parameters, cannibalism in consumer populations can not only monotonically decrease or increase, but also temporarily peak before being eliminated by the mechanisms of mutation and selection.

The complexity of the evolutionary scenarios identified in this study by varying a couple of environmental and demographic parameters, explain why it is difficult to extract a general verbal theory of the evolution of cannibalism from the many available studies performed on different species. However, it is worth to notice that some of the conclusions drawn by Polis in his remarkable papers on cannibalism evolution (Polis, 1981, 1988) are consistent with our findings.

\section{References}

Ascher, U. M. and Spiteri, R. J. 1994. Collocation software for boundary value differential-algebric equations. SIAM Journal on Scientific Computing 15, 938-952.

Byström, P. and Garcia-Berthóu, E. 1999. Density dependent growth and size specific competitive interactions in young fish. Oikos 86, 217-232.

Champagnat, N., Ferrière, R., and Ben Arous, G. 2001. The canonical equation of adaptive dynamics: a mathematical view. Selection 2,73-83.

Claessen, D., de Roos, A. M., and Persson, L. 2000. Dwarfs and giants: Cannibalism and competition in size-structured populations. The American Naturalist 155, 219-237.

Dieckmann, U. and Law, R. 1996. The dynamical theory of coevolution: A derivation from stochastic ecological processes. Journal of Mathematical Biology 34, 579-612.

Dieckmann, U., Marrow, U., and Law, R. 1995. Evolutionary cycling in predator-prey interactions: Population dynamics and the Red Queen. Journal of Theoretical Biology 176, 91-102.

Doedel, E., Champneys, A., Fairgrieve, T., Kuznetsov, Y. A., Sandstede, B., and Wang, X. 1997. AUTO97: Continuation and bifurcation software for ordinary differential equations (with HOMCONT). Computer Science, Concordia University, Montreal, Canada.

Fox, L. R. 1975. Cannibalism in natural populations. Annual Review of Ecology and Systematics 6, 87-106. 
Geritz, S. A. H., Kisdi, E., van der Meijden, E., and Metz, J. A. J. 1999. Evolutionarily dynamics of seed size and seedling competitive ability. Theoretical Population Biology 55, 324-343.

Geritz, S. A. H., Metz, J. A. J., Kisdi, E., and Meszéna, G. 1997. The dynamics of adaptation and evolutionary branching. Physical Review Letters 78, 2024-2027.

Hofbauer, J. and Sigmund, K. 1990. Adaptive dynamics and evolutionary stability. Mathematical Letters 3, 75-79.

Holcik, J. 1977. Changes in fish community of Kikava Reservoir with particular reference to Eurasian perch. Fisheries Research Board of Canada 34, 1734-1747.

Kuznetsov, Y. A. 1998. Elements of Applied Bifurcation Theory. Springer Verlag, Berlin.

Kuznetsov, Y. A. and Levitin, V. V. 1997. CONTENT: A multiplatform environment for analyzing dynamical systems. Dynam. System Lab., Centrum voor Wiskunde en Inform., Amsterdam, available from ftp.cwi.nl/pub/CONTENT.

Matsuda, H. and Abrams, P. A. 1994a. Runaway evolution to self-extinction under asymmetrical competition. Evolution 48, 1764-1772.

Matsuda, H. and Abrams, P. A. 1994b. Timid consumers: Self extinction due to adaptive change in foraging and anti-predator effort. Theoretical Population Biology 45, 76-91.

Maynard Smith, J. 1982. Evolution and the Theory of Games. Cambridge University Press, Cambridge, UK.

Maynard Smith, J. and Price, J. 1973. The logic of animal conflict. Nature 246, 15-18.

Metz, J. A. J., Nisbet, R. M., and Geritz, S. A. H. 1992. How should we define fitness for general ecological scenarios? Trends in Ecology \& Evolution 7, 198-202.

Polis, G. A. 1981. The evolution and dynamics of intraspecific predation. Annual Review of Ecology and Systematics 12, 225-251.

Polis, G. A. 1988. Exploitation competition and the evolution of interference, cannibalism and intraguild predation in age/size-structured populations. In Size-Structured Populations: Ecology and Evolution (B. Ebenman and L. Persson, eds.). Springer, Heidelberg, pp. 185-202. 


\section{Appendix: Analysis of the monomorphic canonical equation}

In this Appendix we analyze the monomorphic canonical equation $(6,7)$ to show that for suitable values of the parameters three equilibria can exist: a stable ESS $\bar{x}_{1}^{\prime}$, an unstable equilibrium $\bar{x}_{1}^{\prime \prime}$ and a stable branching point $\bar{x}_{1}^{\prime \prime \prime}$.

Define $a_{i j}\left(x_{i}, x_{j}\right)=a_{i}\left(x_{i}\right) \sigma\left(x_{i} / x_{j}\right)$, for $i, j>0$ (see eq. (3)), where

$$
a_{i}\left(x_{i}\right)=A_{i j} \frac{x_{i}^{\gamma}}{\underline{x}^{\gamma}+x_{i}^{\gamma}}\left(1-\frac{x_{i}^{\delta}}{\bar{x}^{\delta}+x_{i}^{\delta}}\right) \quad \sigma(z)=\frac{2}{(p z)^{\beta}+(p z)^{-\beta}}
$$

Thus, from eqs. $(1,5,7)$ it follows that

$$
\begin{aligned}
& \left.\frac{\partial \bar{f}_{2}\left(x_{1}, x_{2}\right)}{\partial x_{2}}\right|_{x_{2}=x_{1}}=\frac{e_{20} \frac{\mathrm{d} a_{10}}{\mathrm{~d} x_{1}} n_{0}+\left.e_{21} \frac{\partial a_{21}}{\partial x_{2}}\right|_{x_{2}=x_{1}} \bar{n}_{1}}{1+h_{1} a_{10} n_{0}+h_{1} a_{11} \bar{n}_{1}} \\
& -\frac{e_{20} a_{10} n_{0}+e_{21} a_{11} \bar{n}_{1}}{\left(1+h_{1} a_{10} n_{0}+h_{1} a_{11} \bar{n}_{1}\right)^{2}} \\
& {\left[\frac{\mathrm{d} h_{1}}{\mathrm{~d} x_{1}}\left(a_{10} n_{0}+a_{11} \bar{n}_{1}\right)+h_{1}\left(\frac{\mathrm{d} a_{10}}{\mathrm{~d} x_{1}} n_{0}+\left.\frac{\partial a_{21}}{\partial x_{2}}\right|_{x_{2}=x_{1}} \bar{n}_{1}\right)\right]} \\
& -\frac{\left.\frac{\partial a_{12}}{\partial x_{2}}\right|_{x_{2}=x_{1}} \bar{n}_{1}}{1+h_{1} a_{10} n_{0}+h_{1} a_{11} \bar{n}_{1}} \\
& \left.\frac{\partial^{2} \bar{f}_{2}\left(x_{1}, x_{2}\right)}{\partial x_{2}^{2}}\right|_{x_{2}=x_{1}}=\frac{e_{20} \frac{\mathrm{d}^{2} a_{10}}{\mathrm{~d} x_{1}^{2}} n_{0}+\left.e_{21} \frac{\partial^{2} a_{21}}{\partial x_{2}^{2}}\right|_{x_{2}=x_{1}} \bar{n}_{1}}{1+h_{1} a_{10} n_{0}+h_{1} a_{11} \bar{n}_{1}} \\
& -2 \frac{e_{20} \frac{\mathrm{d} a_{10}}{\mathrm{~d} x_{1}} n_{0}+\left.e_{21} \frac{\partial a_{21}}{\partial x_{2}}\right|_{x_{2}=x_{1}} \bar{n}_{1}}{\left(1+h_{1} a_{10} n_{0}+h_{1} a_{11} \bar{n}_{1}\right)^{2}} \\
& {\left[\frac{\mathrm{d} h_{1}}{\mathrm{~d} x_{1}}\left(a_{10} n_{0}+a_{11} \bar{n}_{1}\right)+h_{1}\left(\frac{\mathrm{d} a_{10}}{\mathrm{~d} x_{1}} n_{0}+\left.\frac{\partial a_{21}}{\partial x_{2}}\right|_{x_{2}=x_{1}} \bar{n}_{1}\right)\right]} \\
& +2 \frac{e_{20} a_{10} n_{0}+e_{21} a_{11} \bar{n}_{1}}{\left(1+h_{1} a_{10} n_{0}+h_{1} a_{11} \bar{n}_{1}\right)^{3}} \\
& {\left[\frac{\mathrm{d} h_{1}}{\mathrm{~d} x_{1}}\left(a_{10} n_{0}+a_{11} \bar{n}_{1}\right)+h_{1}\left(\frac{\mathrm{d} a_{10}}{\mathrm{~d} x_{1}} n_{0}+\left.\frac{\partial a_{21}}{\partial x_{2}}\right|_{x_{2}=x_{1}} \bar{n}_{1}\right)\right]^{2}} \\
& -\frac{e_{20} a_{10} n_{0}+e_{21} a_{11} \bar{n}_{1}}{\left(1+h_{1} a_{10} n_{0}+h_{1} a_{11} \bar{n}_{1}\right)^{2}} \\
& {\left[\frac{\mathrm{d}^{2} h_{1}}{\mathrm{~d} x_{1}^{2}}\left(a_{10} n_{0}+a_{11} \bar{n}_{1}\right)+2 \frac{\mathrm{d} h_{1}}{\mathrm{~d} x_{1}}\left(\frac{\mathrm{d} a_{10}}{\mathrm{~d} x_{1}} n_{0}+\left.\frac{\partial a_{21}}{\partial x_{2}}\right|_{x_{2}=x_{1}} \bar{n}_{1}\right)\right.} \\
& \left.+h_{1}\left(\frac{\mathrm{d}^{2} a_{10}}{\mathrm{~d} x_{1}^{2}} n_{0}+\left.\frac{\partial^{2} a_{21}}{\partial x_{2}^{2}}\right|_{x_{2}=x_{1}} \bar{n}_{1}\right)\right] \\
& -\frac{\left.\frac{\partial^{2} a_{12}}{\partial x_{2}^{2}}\right|_{x_{2}=x_{1}} \bar{n}_{1}}{1+h_{1} a_{10} n_{0}+h_{1} a_{11} \bar{n}_{1}}
\end{aligned}
$$

where

$$
\begin{gathered}
\left.\frac{\partial a_{i j}}{\partial x_{i}}\right|_{x_{i}=x_{j}}=\frac{\mathrm{d} a_{i}}{\mathrm{~d} x_{i}} \sigma(1)+\left.a_{i} \frac{\mathrm{d} \sigma}{\mathrm{d} z}\right|_{z=1} \frac{1}{x_{j}} \\
\left.\frac{\partial a_{i j}}{\partial x_{j}}\right|_{x_{j}=x_{i}}=-\left.a_{i} \frac{\mathrm{d} \sigma}{\mathrm{d} z}\right|_{z=1} \frac{1}{x_{i}}
\end{gathered}
$$




$$
\begin{gathered}
\left.\frac{\partial^{2} a_{i j}}{\partial x_{i}^{2}}\right|_{x_{i}=x_{j}}=\frac{\mathrm{d}^{2} a_{i}}{\mathrm{~d} x_{i}^{2}} \sigma(1)+\left.2 \frac{\mathrm{d} a_{i}}{\mathrm{~d} x_{i}} \frac{\mathrm{d} \sigma}{\mathrm{d} z}\right|_{z=1} \frac{1}{x_{j}}+\left.a_{i} \frac{\mathrm{d}^{2} \sigma}{\mathrm{d} z^{2}}\right|_{z=1} \frac{1}{x_{j}^{2}} \\
\left.\frac{\partial^{2} a_{i j}}{\partial x_{j}^{2}}\right|_{x_{j}=x_{i}}=a_{i}\left[\left.\frac{\mathrm{d}^{2} \sigma}{\mathrm{d} z^{2}}\right|_{z=1}+\left.2 \frac{\mathrm{d} \sigma}{\mathrm{d} z}\right|_{z=1}\right] \frac{1}{x_{i}^{2}}
\end{gathered}
$$

Then, assume $p=0.2, w_{1}=0.1$ and $w_{2}=0.25$ (as in Figures 2-5). Thus, $\sigma(1), \mathrm{d} \sigma /\left.\mathrm{d} z\right|_{z=1}$ and $\mathrm{d}^{2} \sigma /\left.\mathrm{d} z^{2}\right|_{z=1}$ are positive and small (order $10^{-1}$ ). Moreover, $h_{1} \simeq w_{1}$ for all sufficiently high values of $x_{1}$ (see eq. (4)), so that $\mathrm{d} h_{1} / \mathrm{d} x_{1}$ and $\mathrm{d}^{2} h_{1} / \mathrm{d}^{2} x_{1}$ can be neglected when $x_{1}$ is sufficiently high.

Let us now study the function $\bar{n}_{1}\left(x_{1}\right)$. From eq. (1) with $N=0$ it follows that $\bar{n}_{1}\left(x_{1}\right)$ is the positive root of the second order equation

$$
e_{10} a_{10} n_{0}=c_{11} \bar{n}_{1}\left(1+h_{1} a_{10} n_{0}\right)+\left(1-e_{11}\right) a_{11} \bar{n}_{1}+c_{11} \bar{n}_{1}^{2} h_{1} a_{11}
$$

If $x_{1}<\underline{x}$, then $a_{11} \ll a_{10}$, since $a_{1}\left(x_{1}\right)$ and $\sigma(1)$ are small. Thus, the second and third terms at the righthand side of eq. (A3) can be neglected, i. e.

$$
\bar{n}_{1}\left(x_{1}\right) \simeq \frac{e_{10} a_{10} n_{0}}{c_{11}\left(1+h_{1} a_{10} n_{0}\right)}=\tilde{n}_{1}\left(x_{1}\right)
$$

More precisely, $\tilde{n}_{1}\left(x_{1}\right)$ is greater that $\bar{n}_{1}\left(x_{1}\right)$ for any positive $x_{1}$. Thus, $\bar{n}_{1}\left(x_{1}\right)$ is bell-shaped as $a_{10}$ but peaks at a value of $x_{1}$ greater than $x^{0}$ and increasing with $n_{0}$ (notice that the parameter $A_{10}$ can be scaled to 1 , since it is always multiplied by $n_{0}$ ).

Then, consider eqs. (A1,A2) for $x_{1}$ in a neighborhood of $x^{0}$. Taking into account that $x^{0}<\underline{x}$, that $\sigma(1), \mathrm{d} \sigma /\left.\mathrm{d} z\right|_{z=1}, \mathrm{~d}^{2} \sigma /\left.\mathrm{d} z^{2}\right|_{z=1}$ are small, and using (A4)we can neglect in eqs. (A1,A2) all terms containing $a_{i j}$ and their derivates with $i, j>0$, so that

$$
\begin{gathered}
\left.\frac{\partial \bar{f}_{2}\left(x_{1}, x_{2}\right)}{\partial x_{2}}\right|_{x_{2}=x_{1}} \simeq \frac{e_{20} \frac{\mathrm{d} a_{10}}{\mathrm{~d} x_{1}} n_{0}}{1+h_{1} a_{10} n_{0}}\left(1-\frac{h_{1} a_{10} n_{0}}{1+h_{1} a_{10} n_{0}}\right) \\
\left.\frac{\partial^{2} \bar{f}_{2}\left(x_{1}, x_{2}\right)}{\partial x_{2}^{2}}\right|_{x_{2}=x_{1}} \simeq\left[\frac{e_{20} \frac{\mathrm{d}^{2} a_{10}}{\mathrm{~d} x_{1}^{2}} n_{0}}{1+h_{1} a_{10} n_{0}}-2 \frac{e_{20} h_{1}\left(\frac{\mathrm{d} a_{10}}{\mathrm{~d} x_{1}}\right)^{2} n_{0}^{2}}{\left(1+h_{1} a_{10} n_{0}\right)^{2}}\right]\left(1-\frac{h_{1} a_{10} n_{0}}{1+h_{1} a_{10} n_{0}}\right)
\end{gathered}
$$

For sufficiently small values of $n_{0}$, i. e. when $\left(h_{1} a_{10} n_{0}\right) /\left(1+h_{1} a_{10} n_{0}\right) \ll 1$, the two above expressions can be further simplified to

$$
\left.\left.\frac{\partial \bar{f}_{2}\left(x_{1}, x_{2}\right)}{\partial x_{2}}\right|_{x_{2}=x_{1}} \simeq e_{20} \frac{\mathrm{d} a_{10}}{\mathrm{~d} x_{1}} n_{0} \quad \frac{\partial^{2} \bar{f}_{2}\left(x_{1}, x_{2}\right)}{\partial x_{2}^{2}}\right|_{x_{2}=x_{1}} \simeq e_{20} \frac{\mathrm{d}^{2} a_{10}}{\mathrm{~d} x_{1}^{2}} n_{0}
$$

Since $\mathrm{d} a_{10} /\left.\mathrm{d} x_{1}\right|_{x_{1}=x^{0}}=0$ and $\mathrm{d}^{2} a_{10} /\left.\mathrm{d} x_{1}^{2}\right|_{x_{1}=x^{0}}<0$, the approximations (A5) imply that (for suitable values of the parameters) model $(6,7)$ has a stable ESS at $\bar{x}_{1}^{\prime}$ close to $x^{0}$.

The approximations (A5) do not hold for higher values of $x_{1}$, since for such values $a_{10}$, $\mathrm{d} a_{10} /\left.\mathrm{d} x_{1}\right|_{x_{1}=x^{0}}$ and $\mathrm{d}^{2} a_{10} /\left.\mathrm{d} x_{1}^{2}\right|_{x_{1}=x^{0}}$ are small. In particular, in eq. (A1) $\partial a_{21} /\left.\partial x_{2}\right|_{x_{2}=x_{1}}$ is negative for $x_{1}$ sufficiently high, due to presence of the threshold $\bar{x}$ (see eq. (3)). This can lead to a negative sum of the first two terms in eq. (A1) which can balance the third term which is positive.

By contrast, for intermediate values of $x_{1}$, and in particular for $x_{1}$ close to $\underline{x}, \partial a_{21} /\left.\partial x_{2}\right|_{x_{2}=x_{1}}$ is positive and can give rise to a positive $\partial \bar{f}_{2} /\left.\partial x_{2}\right|_{x_{2}=x_{1}}$. This implies the presence of two other equilibria of model $(6,7)$, namely an unstable equilibrium $\bar{x}_{1}^{\prime \prime}$ and a stable equilibrium $\bar{x}_{1}^{\prime \prime \prime}$. The sign of $\partial^{2} \bar{f}_{2} /\left.\partial x_{2}^{2}\right|_{x_{2}=\bar{x}_{1}^{\prime \prime \prime}}$ is more difficult to assess. However, for the parameter settings used 
throughout the paper, the first term of eq. (A2) is dominant and can be positive when evaluated at $\bar{x}_{1}^{\prime \prime \prime}$, so that $\bar{x}_{1}^{\prime \prime \prime}$ is a branching point for suitable values of the parameters.

Finally, it is worth to remark that qualitative analysis is useful for understanding if a certain phenomenon can occur. For confirming the results achieved through this qualitative analysis we have performed extensive numerical analyses, mainly based on continuation techniques (Kuznetsov and Levitin, 1997; Doedel et al., 1997) (see Figures 3,4). 